https://doi.org/10.21670/ref.1908029

Artículos

\title{
El espectro de ciudades santuario en Estados Unidos: Los contrastes en la génesis y las prácticas de las políticas locales proinmigrantes
}

\author{
The spectrum of sanctuary cities in the United States: \\ Contrasting the genesis and practices of \\ proimmigrant local policies
}

Estefanía Cruz Lera a* (iD http://orcid.org/0000-0002-2769-8483

${ }^{a}$ Universidad Nacional Autónoma de México, Centro de Investigaciones sobre América del Norte, México, correo electrónico: estefania.cruz@e-campus.uab.cat

\section{Resumen}

Las denominadas ciudades santuario son actualmente la principal fuente de oposición a las políticas federales antiinmigrantes en Estados Unidos. El argumento central de este estudio cross caso, es que la categoría santuario aglutina un conjunto de leyes, políticas y prácticas informales de diferente naturaleza, con génesis polí-

Recibido el 6 de marzo de 2019. Aceptado el 7 de junio de 2019. Publicado el 8 de julio de 2019.

${ }^{*}$ Autor para correspondencia: Estefanía Cruz Lera, correo electrónico estefania.cruz@e-campus. uab.cat

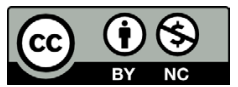

Esta obra está protegida bajo una Licencia Creative Commons Atribución-NoComercial 4.0 Internacional. ticas variadas y con diferentes grados de insurgencia. Este artículo sostiene que el estudio a profundidad del contexto en el que se enmarca cada tipo de santuario puede explicar la existencia de un espectro tan contrastante. Para diferenciarlas se proponen tres categorías representativas dentro del espectro: los santuarios de retórica, los santuarios de facto y los santuarios de bienvenida. Este estudio concluye que las prácticas santuario más representativas — con mayor insurgencia y grado de escalabilidad- están ligadas a la necesidad de la ciudad de defender su acceso a fondos y proteger su autonomía política; pero también, a la capacidad de los migrantes organizados para hacer alianzas con actores políticos locales.

Palabras clave: ciudades santuario, política migratoria, migración local en EE.UU.

\section{Abstract}

The so called sanctuary cities are actually the main source of opposition to anti-immigrant federal policies in the United States. The central argument of this cross case study is that the category of sanctuary agglutinates a set of laws, policies and informal practices of different nature, with varied political genesis and different degrees of insurgency. This article argues that indeep study of the context framing each different kind of sanctuary city helps

CÓMO CITAR: Cruz, E. (2019). El espectro de ciudades santuario en Estados Unidos: Los contrastes en la génesis y las prácticas de las políticas locales proinmigrantes [The spectrum of sanctuary cities in the United States: contrasting the genesis and practices of proimmigrant local policies]. Estudios Fronterizos, 20, e029. doi:https://doi.org/10.21670/ref.1908029 
to explain the existence of a contrasting spectrum. With the aims to contrast them, three representative categories within the spectrum are analyzed: sanctuary of rhetoric, informal sanctuary, and welcoming sanctuary. This research concludes that the most representative sanctuary practices - the most contestative and with the highest degree of scalability - are linked to the need of the city to defend funding access and to protect its political autonomy; but also, they are linked to the capacity of organized migrants to make alliances with local political stakeholders.

Keywords: sanctuary cities, immigration policy, US local immigration.

\section{Introducción}

Los inmigrantes constituyen aproximadamente $16.9 \%$ de la fuerza laboral en Estados Unidos y se estima que $63 \%$ de ellos están concentrados en solamente veinte zonas metropolitanas (Passel y Cohn, 2017). La mayor parte de estas ciudades son destinos tradicionales que han construido narrativas como hito fundacional alrededor de la migración para agilizar su incorporación a las dinámicas urbanas (Hoekstra, 2017). Las economías urbanas atraen a más trabajadores extranjeros y además son la puerta de entrada a otras áreas del país. Estas dinámicas generan respuestas locales altamente contrastantes hacia los inmigrantes.

En Estados Unidos, la Constitución delega todos los poderes sobre control de aduanas y fronteras al gobierno federal (política migratoria), mientras que los gobiernos locales son incentivados a crear políticas de acogida e integración (política de inmigración). En este contexto, las ciudades encuentran un nicho político en la paradoja de que la Constitución de Estados Unidos ordena tanto que el gobierno federal es responsable de mantener estricto control fronterizo, como que cada individuo en el país tiene derechos sin importar su origen nacional o estatus migratorio (Chand y Schreckhise, 2015; Varsanyi, Lewis, Provine y Decker, 2011). Esta paradoja entre un régimen de ciudadanía excluyente y los estándares constitucionales de personalidad, debido proceso y garantías individuales, han ampliado las posibilidades para que los gobiernos locales encuentren mecanismos institucionales para proteger a su población indocumentada de la deportación basada exclusivamente en la falta de documentos migratorios.

En este contexto de incremento de respuestas locales para proteger a los migrantes indocumentados de la política federal antiinmigrante, surgen las siguientes interrogantes que guían esta investigación:

- ¿Qué motiva a los gobiernos locales para oponerse a las medidas antiinmigrantes de los estados y del gobierno federal?

- ¿Qué contrastes y similitudes se presentan en las políticas locales de inmigración?

- ¿Qué variables explican su grado de insurgencia frente a la política federal? 
Para resolver estas interrogantes, esta investigación se enfoca en el análisis de las llamadas ciudades santuario. El punto de partida es que no hay una definición oficial de qué son las ciudades santuario, este es un término que aglutina desde ciudades con prácticas no oficiales de no cooperación con los oficiales migratorios, hasta el extremo contrario de ciudades con leyes, ordenanzas y políticas concretas de acogida (Gonzalez, Collingwood y El-Khatib, 2017).

Esta categorización nos lleva a plantear la primera hipótesis de trabajo:

H1 El movimiento santuario aglutina a un conjunto heterogéneo de prácticas, leyes y políticas que comparten su motivación originaria pero que difieren en sus efectos prácticos.

Es decir, esta investigación sostiene que no todas las leyes y políticas santuario tienen efectos concretos sobre la gestión migratoria local y sobre las vidas de los inmigrantes indocumentados. Las leyes santuario incorporan mecanismos de distensión del reforzamiento migratorio para hacer frente a contextos políticos adversos hacia sus inmigrantes. Por otro lado, las prácticas santuario no son exclusivamente oficiales y se orientan a la atención social de migrantes más vulnerables.

Varios organismos han creado listas y mapas de ciudades santuario, destacan la lista del Departamento de Seguridad Interior (2017), el del Immigrant Legal Resource Center (2018), y el del Center for Immigration Studies (2019). El problema es que estos mapas no exploran cada caso a profundidad y menos toman en cuenta su contexto concreto ni su génesis específica.

El Departamento de Justicia de Estados Unidos enlista en su base de datos a 47 ciudades consideradas santuario (The United States Department of Justice [USDJ], 2017). La lista incluye grandes metrópolis como Nueva York y Los Ángeles, pero también pequeños pueblos como Winooski y Aberdeen. En contraste, otras ciudades con las tasas más altas de población nacida en el extranjero como Hialeah $(74.4 \%)$ y Miami $(56.4 \%)$ en Florida no son santuarios. Esta paradoja conduce a formular una segunda hipótesis:

H2 La tasa de inmigrantes en la ciudad no determina la adopción de políticas proinmigrantes como sí lo hace su capital sociopolítico en la localidad.

Lieberman (2013) explica que una estructura institucional fragmentada y descentralizada ofrece múltiples puntos de acceso para los migrantes que buscan influencia sobre las arenas políticas locales. Sin embargo, esta misma descentralización produce contrastes entre las respuestas de los gobiernos locales, lo cual puede fungir como un arma de doble filo para los inmigrantes, sobre todo cuando estas respuestas erosionan las discusiones sobre cambios profundos y estructurales en las leyes y políticas migratorias.

Tomando en cuenta las variaciones entre una y otra ciudad, es poco práctico establecer categorías analíticas rígidas y resulta más fructífero estudiar a profundidad los casos con mayor grado de innovación política. En este sentido, la tercera hipótesis de trabajo es:

H3 El análisis de las trayectorias políticas permitirá identificar diferentes procesos de génesis, diversos enfoques y variados grados de insurgencia dentro del espectro de las ciudades santuario.

Hay un creciente reconocimiento de que las ciudades juegan un papel cada vez más importante sobre la gestión migratoria. Al respecto se han hecho numerosos estudios de caso a profundidad sobre la gestión local de la inmigración; sin embargo, son escasos los estudios enfocados en las variaciones y contrastes de estas respuestas 
locales (Schiller, Çaglar y Guldbrandsen, 2006). Con la motivación de contribuir a este llamado, esta investigación hace un estudio cross-caso de las ciudades santuario con mayor influencia política en el contexto actual.

\section{Datos y métodos}

El diseño de investigación por la naturaleza del tema y de las preguntas que motivan el estudio fue inductivo y cualitativo. En su conjunto los datos de esta investigación fueron reunidos a través de una estrategia multimetodológica.

En este trabajo se recuperan los datos obtenidos durante el trabajo de campo para distintas investigaciones llevadas a cabo entre 2014 y 2018 sobre la incorporación política de migrantes en los estados de California, Texas e Illinois, así como en las ciudades de Filadelfia y Nueva York. Entre los resultados del trabajo empírico en estas ciudades santuario se encontró que hay una retroalimentación entre las políticas del lugar y la agencia de los actores políticos locales. En cada caso, las ciudades santuario fueron un contexto de partida que apalancó las dinámicas políticas de los inmigrantes en la localidad; sin embargo, la protección que ofrecen oscila entre el discurso, el procedimiento y en menor medida los programas públicos.

El trabajo de campo incluyó la observación participante en las reuniones de los inmigrantes con los gobiernos locales, en las conferencias de las autoridades y en las demostraciones públicas de las organizaciones. En los casos concretos de California y Chicago se incluyen fragmentos de entrevistas realizadas con autoridades locales y activistas migrantes. Posteriormente, en la fase de documentación de los casos, también se hizo una recolección de reportes de organizaciones de la sociedad civil, así como el seguimiento de medios de comunicación impresos y televisivos sobre las legislaciones y programas de las ciudades.

Finalmente, y reconociendo que las prácticas y políticas santuario en casi todos los casos son anteriores a su institucionalización a través de las leyes, se recurrió a las fuentes primarias. La Tabla 1 enlista las ordenanzas y leyes analizadas:

La selección de casos para ejemplificar cada categoría se basó en dos criterios, el primero relacionado con reducir la ambigüedad del término santuario, y el segundo fue el grado de innovación del caso dentro de cada categoría.

Para el análisis de datos se utilizó la técnica conocida como análisis de trayectorias políticas, englobada en el cuerpo metodológico de rastreo de procesos cualitativos, este enfoque transaccional busca los mecanismos causales y las dinámicas concretas que derivaron en un proceso político influyendo sobre cauce. Es decir, en orden cronológico inverso se rastrea la historia concreta y las interacciones entre los actores involucrados para reconstruir la trayectoria política de los casos identificados como representativos.

\section{Aproximaciones teóricas al estudio de las ciudades santuario}

La ciudad, como escala de análisis, ofrece un escenario muy fructífero para estudiar las dinámicas políticas derivadas de la migración. En las ciudades la diversidad y el cambio étnico-demográfico son procesos mucho más visibles. El contexto local 
permite enfocarnos en las circunstancias políticamente relevantes para analizar la responsividad del sistema político, en particular, la forma en que los intereses y los valores se acomodan en la esfera pública local (Glick y Caglar, 2009). Los gobiernos locales frecuentemente se ven orillados a tener que mediar las diferentes agendas y posturas entre sus poblaciones nativas y sus poblaciones inmigrantes. Este proceso de acomodación da como resultado políticas de inclusión o exclusión dependiendo de las características endógenas de cada localidad.

Tabla 1. Lista de leyes santuario revisadas

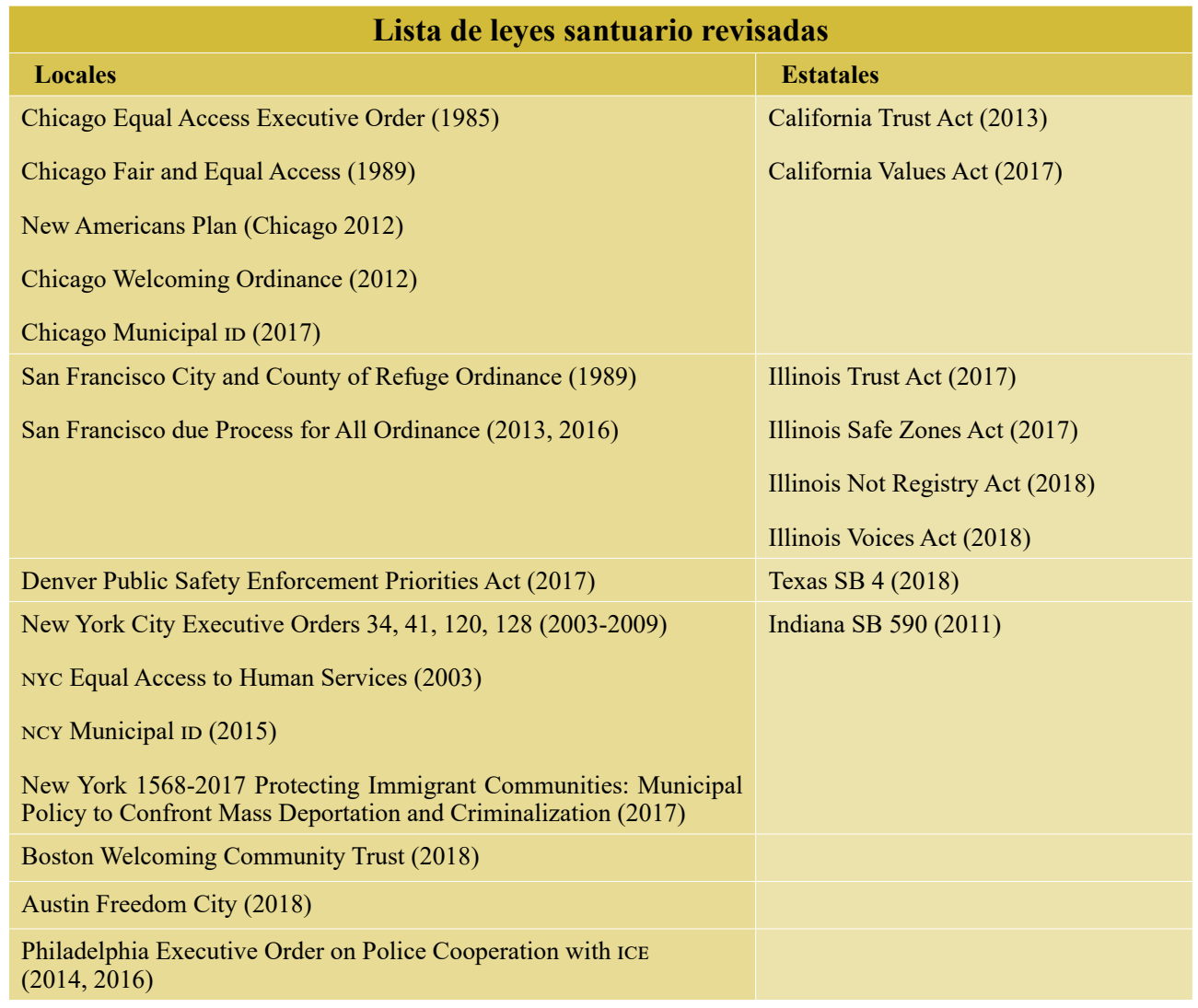

Fuente: Elaboración propia con datos del trabajo empírico.

En las urbes las posibilidades de acción colectiva, los servicios ofrecidos por la sociedad civil organizada, el acceso a recursos y los mecanismos de participación en la arena pública son mayores y están más al alcance de los inmigrantes. Mollenkopf sostiene que enfocarse en la escala local permite descifrar la construcción y el mantenimiento de coaliciones locales, elucidando las tensiones entre ellos que podrían causar tanto rupturas como reorganización, especialmente cuando descansan en parte en la movilización de migrantes (Mollenkopf, 2013, p. 114).

Las diferentes vías de los gobiernos locales en su gestión migratoria también han dado lugar a varias líneas de estudio. Sin duda, la línea más nutrida corresponde a las respuestas negativas, la oposición local y las políticas de exclusión (Harwood y Myers, 2002; Hopkins, 2010; Varsanyi, 2011). Esta tendencia se repite en la documentación 
periodística, las respuestas antiinmigrantes despiertan más interés de la prensa y la opinión pública que las buenas prácticas.

En la literatura anglosajona se han desarrollado numerosos estudios y reportes sobre la relación entre la gestión local de la inmigración y el crimen. Kent y Carmichael (2017) encontraron en su estudio que a mayor segregación racial y mayores niveles de desempleo en la ciudad, es más probable que la policía apoye actividades de reforzamiento migratorio. En cuanto a la relación santuario-crimen, Lyons, Velez y Santoro (2013) descubrieron que, a diferencia de lo que los opositores de las políticas santuario citan, las ciudades de su muestra tienen menores tasas de crímenes violentos cometidos por migrantes indocumentados.

La faceta legalista de las ciudades santuario también ha sido ampliamente abordada, principalmente en torno a los derechos de las localidades para litigar por su autonomía y en defensa del acceso a fondos federales. Destacan los estudios sobre procesos de devolución federal y descentralización que analizan las facultades de los gobiernos locales para legislar sobre inmigración y para restringir la cooperación policiaca en el reforzamiento migratorio (Eisinger, 1998; Varsanyi et al., 2011). También en la línea legalista encontramos estudios con enfoques integracionistas (Chand y Schreckhise, 2015; Cunningham, 2017), aquellos que critican el mosaico de autoridades que se solapan en la política migratoria y pugnan por la unificación de los mecanismos y canales de cooperación legales de los diferentes niveles de gobierno involucrados en la gestión migratoria.

Las leyes y ordenanzas que restringen la cooperación local en el reforzamiento migratorio solo tienen efecto sobre las vidas de los inmigrantes cuando van acompañadas de políticas, programas instrumentales y presupuesto justo. Cornelius (2010) explica que los inmigrantes traen consigo desafíos y oportunidades para las localidades, mismas arenas políticas que pueden tener influencia sobre las políticas migratorias nacionales reforzando o limitando sus efectos. Bajo este supuesto, hay un creciente optimismo de que las ciudades pueden mediar, detener o revertir los efectos de la política migratoria nacional (Filomeno, 2017). Esto ha expandido los horizontes del estudio de las ciudades santuario como un semillero de políticas y prácticas comunitarias relacionadas con la regeneración urbana (Harwood y Myers, 2002; Levine y Gershenson, 2014; Paik, 2017).

Uno de los mayores retos de las investigaciones sobre las ciudades santuario, ha sido demostrar que las dinámicas urbanas no solo tienen efectos locales y que además no están constreñidas exclusivamente por las dinámicas de la ciudad. Bauder (2016) explica que convertirse en una ciudad santuario se equipara más a un proceso político que a un objetivo por decreto. En cambio, el objetivo es ilustrar las diferencias sobre cómo los migrantes y los refugiados son tratados en la localidad y a escala nacional. En la misma dirección, Ridgley (2008) sostiene que las ciudades santuario tal y como existen hoy deben su existencia a las dinámicas de criminalización de la migración.

De acuerdo con Huang y Liu (2016), las necesidades locales y los imperativos económicos unidos a los factores políticos ayudan a explicar por qué algunas ciudades adoptan legislaciones proinmigrantes. Estos autores desarrollaron un modelo de análisis basado en la regresión logística para probar que la tasa de población nacida en el extranjero, las tasas de desempleo y pobreza, la centralidad de la ciudad y, las preferencias electorales son variables diferenciadas entre las ciudades santuario y las que no han presentado este tipo de movimiento. Otro estudio similar fue desarrollado por Ramakrishnan y Wong (2007), quienes sugieren que la adopción de políticas 
proinmigrantes está influenciada por el impacto del cambio demográfico causado por la migración, que progresivamente aumenta la influencia de estos sectores en la política local.

Los estudios anteriores explican las motivaciones y razones de las ciudades para sumarse al movimiento santuario. En contraste, este estudio contribuye a la literatura al explicar los diferentes procesos de génesis y las variaciones en el grado de insurgencia local frente al contexto antiinmigrante federal.

\section{Nodos de continuidad y cambio en el llamado movimiento santuario}

El movimiento santuario en Estados Unidos data de la década de 1980, cuando el gobierno federal declara la terminación del TPS $^{1}$ para los refugiados de guerras civiles de Centroamérica. El fin del reconocimiento de este estatus de protección complementaria vino acompañado de redadas masivas y deportaciones de migrantes de baja prioridad (Gonzalez et al., 2017). Esta política antiinmigrante dio lugar a un movimiento santuario desarrollado por grupos religiosos cuya práctica principal consistió en proveer refugio al interior de infraestructura religiosa a algunos inmigrantes en riesgo inminente de deportación. Esta práctica abogaba por que los templos religiosos al ser espacios reconocidos como socialmente sensibles evitarían que los oficiales de inmigración hicieran efectiva la detención.

La ciudades de Tucson, Chicago, Los Ángeles y otras localidades en Arizona, Texas y California fueron clave en este primer movimiento santuario (Stoltz, Hamilton y Loucky, 2009). En esta etapa, la mayoría de las personas que se refugiaron en las iglesias fueron principalmente padres de familias de estatus mixto. La situación se agudizó en 1986 con las restricciones impuestas por la aprobación de la Ley de Control y Reforma Migratoria (IRCA). Posteriormente, en la década de 1990 el movimiento santuario pierde fuerza con la amnistía y las campañas de regularización migratoria.

En el siglo xxi, el movimiento santuario se reactivó para hacer frente al Acta Patriota (United States Congress, 2001) que asociaba las medidas antiinmigrantes con la seguridad nacional. En esta coyuntura histórica, el movimiento santuario reúne el apoyo de un centenar de templos a lo largo del país, el apoyo de organizaciones civiles, e incluso el compromiso de 47 congresistas (Bilke, 2009). Otra iniciativa durante esta etapa fue el "campus santuario". Movimiento protagonizado por académicos, personal y alumnos de dos centenares de universidades y colegios superiores quienes exigieron la protección dentro del campus de sus estudiantes indocumentados.

El dominio del enfoque restrictivo en migración dio lugar a múltiples episodios de deportaciones de inmigrantes de baja prioridad. Muchos casos movilizaron la opinión pública al atrincherarse en iglesias para evitar la deportaciones (Paik, 2017). Uno de los más famosos es el de la activista comunitaria Elvira Arellano, quien en compañía de su hijo nacido en Estados Unidos, logró permanecer refugiada en una iglesia metodista de Humboldt Park en Chicago durante un año (Cook, 2013). El caso terminó en

${ }^{1}$ El Estatus de Protección Temporal (TPS por sus siglas en inglés) es una visa temporal para una lista de nacionalidades concretas elegibles que no pueden retornar a su país por un conflicto militar en curso, un desastre natural, u otra condición extraordinaria (United States Citizenship and Immigration Services, 2019). 
deportación, pero Arellano logró regresar a EE. UU. con una visa humanitaria para continuar su activismo.

En este punto de la historia cabe resaltar los nodos de continuidad y de ruptura entre el viejo movimiento santuario y el movimiento contemporáneo. El movimiento santuario original estaba orientado hacia los refugiados centroamericanos, tenía una alta connotación moralista de los grupos de fe y se encuadraba dentro los enfoques compasivos hacia la migración (Bender y Arrocha, 2017). En contraste, el denominado "nuevo movimiento santuario" está protagonizado por las ciudades (los gobiernos locales) y su motivación principal es contrarrestar la criminalización de sus residentes por el solo hecho de no tener estatus migratorio formal.

Steil y Vasi (2014) describen cómo durante el 2006 hubo una proliferación de leyes de exclusión que buscaban el refuerzo migratorio a través de la disuasión. Estos autores destacan legislaciones como el uso forzoso del programa E-Verify en los negocios locales, políticas de "solo inglés" en los servicios de la ciudad, restricciones para los no ciudadanos, entre otras medidas destinadas a desmotivar el asentamiento de migrantes indocumentados en las ciudades. Ante esto, las organizaciones de migrantes y las ligas de defensa se movilizaron para presionar a los gobiernos locales de adoptar ordenanzas santuario.

Aunado a las leyes locales antiinmigrantes, entre 2008 y 2014 estuvo vigente el programa Comunidades Seguras (S-Comm), que fue un importante impulsor de las ciudades santuario durante el periodo presidencial de Barack Obama. S-Comm requería a las policías de la ciudad detener a los inmigrantes indocumentados enlistados en las bases de datos de ICE (Oficiales de Inmigración y Aduanas). La principal justificación fue la reducción del crimen; sin embargo, las estadísticas demuestran que alrededor de $50 \%$ de los inmigrantes detenidos mediante este programa no tenían registro criminal previo (Chand y Schreckhise, 2015). Las prácticas de S-Comm, poco claras y muy cuestionadas a nivel comunitario, ocasionaron que se multiplicaran las legislaciones y políticas santuario.

Frecuentemente se cita a San Francisco como la primera ciudad santuario debido a que en 1985 pasó la resolución "City and County of Refuge":

Los empleados federales, no los de la ciudad, deben considerarse como los responsables de la implementación de la política migratoria y de refugio, y los departamentos de la ciudad no deben conducirse de forma alguna que pudiera causar la deportación de refugiados salvadoreños y guatemaltecos. ${ }^{2}$

Sin embargo, fue hasta 1989 que la ciudad adoptó la política de prohibir a los oficiales preguntar por el estatus migratorio o divulgar esta información sensible sobre los residentes de la ciudad. Si bien San Francisco no fue la primera ciudad santuario, sí es la más famosa desde el caso Steinle, ${ }^{3}$ pues a partir de esta situación se relacionó a las ciudades santuario con el crimen.

\footnotetext{
${ }^{2}$ De hecho, es la primera vez que la prensa estadunidense denomina a una ciudad santuario, aunque señalan que anteriormente Los Ángeles, Berkeley, Chicago, San Paul y Cambridge habían firmado resoluciones similares como consta en el archivo del New York Times ("San Francisco Approves", 1985).

${ }^{3}$ Este caso alcanzó atención mediática a nivel nacional porque el asesino de Kate Steinle es un inmigrante indocumentado que tenía récord criminal y nunca fue reportado con la oficina de inmigración y aduanas debido a las ordenanzas santuario de San Francisco. Este caso ha sido usado por los opositores a las ciudades santuario, entre ellos el presidente Trump, incluso los familiares de la víctima fueron invitados de honor en el discurso del Estado de la Unión 2019 para justificar su retórica antiinmigrante.
} 
Otras ciudades como Nueva York, Chicago, Boston y Los Ángeles adoptaron políticas santuarios más fuertes y con antelación. Por ejemplo, Gonzalez et al. (2017) documentan cómo en 1983, la ciudad de Madison en Wisconsin pasó la Resolución 39/105 en la que respaldaba a las iglesias que ofrecían santuario a los refugiados centroamericanos. Fue en 1985 que la ciudad pasó la Resolución 41/075 para limitar la cooperación local con actividades de refuerzo migratorio.

También en 1985, el alcalde de Chicago Harold Washington emitió una orden ejecutiva como resultado de una promesa de campaña para lograr una alianza política entre Afroamericanos y Latinos que le permitió ocupar la alcaldía. La ordenanza de Chicago reducía la cooperación con los oficiales de inmigración y prohibía a los servidores públicos de la ciudad preguntar por el estatus migratorio para solicitar trabajos, licencias y asistencia social. En 1989, el alcalde Daley extendió la ordenanza incluyendo una cláusula de "acceso justo e igualitario" a los servicios de la ciudad de Chicago sin importar el estatus migratorio de sus residentes.

En la actualidad el espectro es cada vez más amplio, las políticas santuario incluyen importantes variaciones. Estas ciudades se han vuelto la principal y más poderosa fuente de oposición en el paradójico escenario de un sistema político donde, por un lado, la política migratoria está bajo la jurisdicción de un gobierno federal antiinmigrante, mientras que por otro, los gobiernos locales son responsables de las políticas de acomodación y los procesos de asimilación en contextos más progresistas. Cabe mencionar que el futuro de las ciudades santuario es incierto, pues sus opositores, entre ellos el poder ejecutivo, están buscando mecanismos legales para penalizar y restringir el impacto de las políticas santuario.

El presidente Donald Trump las ha utilizado como blanco principal de su discurso antiinmigrante, e incluso ha buscado formas para recortar la transferencia de fondos federales a estas ciudades. Por ejemplo, ha utilizado las Becas Byrne que son transferencias de fondos a las ciudades para combatir el crimen violento a las ciudades que están en la citada base de datos de ICE. Aún en este contexto adverso, las ciudades no han dejado de buscar formas de proteger su vitalidad económica y social producto de la migración.

\section{Las ciudades santuario en la actualidad: Tres casos representativos del espectro}

El espectro de ciudades santuario incorpora prácticas, leyes y políticas de distinto orden y naturaleza que resulta complicado clasificarlas mediante categorías rígidas. Sin embargo, hay ciudades muy representativas que destacan entre el microuniverso y cuyos casos permiten analizar su diferente génesis, variada instrumentación y diversos grados de insurgencia local frente al contexto federal antiinmigrante. En este apartado se analizan tres casos representativos: los santuarios de retórica, los santuarios de facto y los santuarios de acogida.

En primer lugar, los santuarios de retórica se caracterizan por tener ordenanzas y leyes proinmigrante que permanecen meramente en un plano discursivo y carecen de mecanismos concretos de instrumentalización. El problema principal de este tipo de prácticas es que las ordenanzas son fácilmente manipulables y modificables por los gobiernos locales, viéndose condicionados a la postura del grupo en el poder frente a 
los inmigrantes. Aunado a lo anterior, la declaración de santuario se hace desvinculada de los grupos de activistas proinmigrantes y sus acciones comunitarias para hacer frente a las restricciones migratorias.

Los críticos de las ciudades santuario explican que incluso cuando hay marcos legales que establecen la no cooperación con las medidas de reforzamiento migratorio, estas pueden llegar a constituir solo un conjunto de buenas intenciones sin mecanismos concretos de instrumentalización. Estos sectores señalan que una ley cuando no forma parte de un conjunto de políticas se convierte en letra muerta, y además una política sin un presupuesto adecuado tiene efectos discursivos solamente. Al respecto, un concejal declaró:

Para mí, es muy importante garantizar que Chicago sea verdaderamente una ciudad santuario, no sólo un santuario de retórica, porque no se pueden pelear políticas antiinmigrantes con retórica proinmigrantes, se tiene que pelear con políticas proinmigrante (Ramírez-Rosa, 2017).

Existen dos escenarios que provocan el surgimiento de santuarios de retórica. El primero se relaciona con ciudades que pasan resoluciones en las que prohíben la cooperación en operativos antiinmigrantes, situación que se presenta esporádicamente debido a sus bajas tasas de inmigrantes. La adopción de medidas santuario se utiliza en estos casos como un recurso político para poner de manifiesto el carácter progresista de la política local. Lo anterior debido a que los episodios de redadas y deportaciones masivas no se presentan o se dan de manera esporádica. Otra situación que motiva la adopción de santuarios de retórica por parte de los gobiernos locales se deriva de la inminente escalada de posiciones antiinmigrantes en los condados cercanos a la metrópolis o en otras ciudades dentro del estado. Algunas de ellas como Denver que no están en el top de ciudades con más inmigrantes se han catalogado como santuario debido a que se localizan en estados con políticas antinmigrantes o cercanas a ciudades con legislaciones altamente restrictivas hacia los residentes indocumentados. El caso de Denver es particularmente interesante en esta categoría de santuarios de retórica.

Denver en Colorado, es reconocida como un importante escenario del movimiento chicano de la década de $1960 .{ }^{4}$ Sin embargo, la historia contemporánea del activismo migrante en esta ciudad difiere mucho de su activo pasado. Se estima que $37 \%$ de los 55000 de sus migrantes son indocumentados (Passel y Cohn, 2017). En 2006, los inmigrantes de Denver se enfrentaron al incremento de leyes antinmigrantes en el estado de Colorado, incluyendo medidas extremas de persecución como la conformación de una patrulla estatal de refuerzo migratorio. Entre 2006 y 2012 estuvo vigente en el estado la Colorado Immigration Reporting Law que consistía en recoger y reportar el estatus migratorio de cualquier persona que tuviera contacto con la policía.

En la ciudad de Denver, las posturas en torno a la migración siempre han estado ampliamente divididas, incluso en 2011 la ciudad formó parte del programa de Comunidades Seguras. Sin embargo, fue en el año 2012 que en el marco de las elecciones mayorales, el Instituto Fiscal de Colorado reportó que el reforzamiento

\footnotetext{
${ }^{4}$ El activista Rodolfo "Corky" González, uno de los líderes más importantes del movimiento chicano nació allí y fundó la Chicano Youth Conference para exigir apoyo a las comunidades mexicanoamericanas de la ciudad.
} 
migratorio en la ciudad estaba afectando la economía y la vitalidad local (Partnership for a New American Economy, 2016) ${ }^{5}$ Ese año el alcalde reelecto Hancock abandonó el programa federal de Comunidades Seguras. Es hasta 2017 que en el consejo de la ciudad se introduce una iniciativa santuario. Entre los principales opositores estaba el Sheriff de la ciudad, que citaba los argumentos que asocian la migración y el crimen. Finalmente, se llega a un acuerdo en agosto de 2017 con una ley bastante enmendada en comparación con la iniciativa original.

Denver es un santuario de retórica debido a que la Public Safety Enforcement Priorities Act (2017) solamente limita la cooperación con ICE en deportaciones y hay numerosas excepciones todas relacionadas con la seguridad pública. Por ejemplo, una de las excepciones incluidas es que las cárceles locales informan a las autoridades migratorias con 24 horas de antelación cuando un inmigrante indocumentado va a ser liberado. Es decir, el espíritu de las ordenanzas santuario de la ciudad contrastan con la forma en que el gobierno local ha priorizado la seguridad pública sobre la inmigración, pues como se ha explicado anteriormente para que haya efectos y no solo cambio discursivo, la relación entre comunidades de estatus mixto y la policía local debe reformularse con enfoques comunitarios. La ordenanza de Denver se queda en el plano discursivo en parte debido a que la comunidad migrante ha desarrollado iniciativas aisladas como las redes de respuesta rápida. ${ }^{6}$ Las organizaciones locales de migrantes son principalmente de carácter cultural y cívico, en su lugar la influencia política se da a través de las ramas locales de ligas de defensa y organizaciones nacionales.

El segundo tipo que se analiza en este artículo son los santuarios de facto, esta dinámica se da cuando una metrópolis con tasas altas de inmigrantes se encuentra en un estado con leyes restrictivas y posturas antiinmigrantes. En estos casos, las ciudades adoptan prácticas informales que van desde no ejecutar la ley estatal, litigar en las cortes a favor de su autonomía en materia de gestión migratoria e incluso llevar a cabo políticas santuario bajo otros nombres o en el marco de otras iniciativas de reestructuración urbana.

En esta situación se encuentra San Antonio en Texas, esta ciudad ha capturado atención mediática por representar el más claro ejemplo de divergencia local-estatal, aunque en la práctica es solo un santuario de retórica debido a la informalidad de sus por demás delimitadas prácticas.

El peso político de los latinos en el bastión republicano de Texas es cada vez más visible. Cuestiones como la juventud y la baja tasa de ciudadanización de los latinos hace que el capital colectivo de esta minoría se incremente progresivamente. En Texas, debido a su condición de estado fronterizo, la migración irregular es un tema altamente divisivo, el estado republicano se ha caracterizado por su postura de apoyo al refuerzo migratorio. Sin embargo, Houston, Dallas y San Francisco están dentro de las 12 áreas metropolitanas con más inmigrantes indocumentados (Passel

\footnotetext{
${ }^{5}$ Se explica que los inmigrantes en Denver representan más de $20 \%$ de la fuerza laboral en industrias locales como la construcción, el transporte, la educación y la recreación. Además expresan que contribuyen con mil millones de dólares cada año a los servicios de salud, mismos que muchos no pueden utilizar por la falta de documentos.

${ }^{6}$ Las redes de respuesta rápida son iniciativas comunitarias que consisten en redes de voluntarios que van a los lugares donde se reporta que hay una redada o detenciones de ICE para vigilar el debido proceso y el respeto a los derechos fundamentales de los inmigrantes.
} 
y Cohn, 2017). Esta circunstancia provoca un conflicto local-estatal en la gobernanza migratoria donde el caso de mayor oposición a nivel local es San Antonio.

San Antonio tiene una práctica informal de no cooperación en actividades de refuerzo migratorio. Si bien no existe una ordenanza local, sí hay una política que incluye la práctica de no cuestionar ni divulgar el estatus migratorio de los detenidos por la policía local. Estas medidas han levantado muchas críticas. En 2017, en el Senado de Texas se pasó la llamada Ley Antisantuario (Texas Senate Bill 4, 2016) que requiere a las ciudades el nombramiento de personal específico para el refuerzo migratorio y la cooperación con ICE, también obliga a la policía local a mantener detenidos a todos aquellos sospechosos de situación migratoria irregular hasta que se confirme esta información. La policía de San Antonio se opuso a esta medida y como respuesta el estado de Texas demandó a la ciudad. En respuesta, El Paso, San Antonio, Austin y Dallas introdujeron una querella en una corte federal para que el asunto se resuelva en los tribunales.

El fiscal del estado declaró en conferencia de prensa:

Desafortunadamente, algunos municipios como San Antonio ponen en riesgo la seguridad de los policías y de los ciudadanos al desafiar la ley estatal. El tribunal debe aceptar nuestra solicitud de interdictos y sanciones civiles contra San Antonio para enviar un mensaje a todas las ciudades de Texas sobre que deben obedecer la ley (Paxton, 2017).

Cabe mencionar que esta ley estatal antiinmigrante establece que los oficiales no electos de la ciudad serán multados por aplicar o difundir las medidas santuario.

En respuesta, el congresista Saldaña en su campaña de activismo contra esta ley explicó:

Ya veremos como la Ley 4 del Senado, la más draconiana ley antiinmigrante del país, resiste a las ciudades texanas listas para defender a sus comunidades y a los más arduos litigadores de la nación del Fondo Mexicano Americano para la Educación y la Defensa Legal (Saldaña, 2018).

Cabe mencionar que los oficiales electos de San Antonio hablan de santuario en la esfera pública fuera de las instituciones, pero la ciudad no tiene ordenanzas santuario oficiales. Es decir, aunque en San Antonio la migración está en la esfera pública, no hay políticas concretas, lo cual implica que solo puede ser considerado un santuario de retórica.

El tercer caso dentro del espectro santuario se refiere a aquellas ciudades cuyas ordenanzas santuario han sido complementadas por políticas de acomodación y que se sustentan en la colaboración entre oficinas públicas y migrantes organizados. Estas ciudades consideran que las ciudades santuario pueden ser impulsoras del cambio político cuando van más allá de la iniciativa "no pregunto, no comparto estatus migratorio". Una activista de Chicago explicó:

Ves como todo el mundo habla de cómo tener mejores ciudades santuario. Pero eso no es una solución a todos los problemas de los inmigrantes. Sabemos que los santuarios son una herramienta que estamos utilizando para prevenir y resistir. Pero esa es la clave, prevenir que los recursos públicos sean utilizados para financiar deportaciones en lugar de ser invertidos en el desarrollo urbano (activista I, 2017). 
Dada la ambigüedad del término ciudades santuario y su criminalización por sus opositores al llamarlas "semilleros de delincuentes" (Sessions, 2017), las ciudades han buscado formas para que sus legislaciones y políticas no sean encasilladas en clasificaciones rígidas como las del Departamento de Seguridad Interior y el Departamento de Justicia. Así, encontramos que las ciudades con ordenanzas más progresistas tienden a crear welcoming cities (ciudades de bienvenida), compassionate cities (ciudades de compasión), freedom cities (ciudades de libertad), todas ellas expansiones de las ordenanzas santuario.

Un caso muy representativo del desarrollo de estos santuarios de acogida es el de Chicago ciudad de bienvenida. Un aspecto a considerar de "la ciudad que trabaja" es como esta ciudad de inmigrantes ha logrado mantener sus políticas de bienvenida a pesar de que se encuentra aislada en un estado republicano en el Medio Oeste rural. Chicago es una de las ciudades que ha protagonizado la defensa y la expansión de sus políticas santuario en respuesta a la política antiinmigrante del presidente Trump.

En 2011 cuando Rahm Emmanuel fue elegido como alcalde, los líderes migrantes pensaron que esto podría erosionar la relación entre sus organizaciones y el ayuntamiento. Emmanuel no fue un abogado de la reforma migratoria cuando trabajó en la Casa Blanca con Barack Obama y esto causaba desconfianza entre la comunidad migrante. Sin embargo, siendo los latinos una importante fuerza política local, el alcalde tenía que responder a las demandas por expandir las medidas del viejo movimiento santuario en la ciudad.

En 2012, en Chicago se aprobó el New Americans Plan (2012) y las Welcoming Ordinances (2012) que en conjunto ayudaban a extender todos los servicios ofrecidos por la ciudad a sus residentes indocumentados. Así, la ciudad de Chicago desarrolló programas de asistencia legal, cursos de inglés como segundo lenguaje, incubadoras de negocios para inmigrantes y otros programas públicos para la incorporación de los migrantes sin importar su estatus migratorio. Aunado a lo anterior, Chicago lanzó junto a Nueva York, Boston y Los Ángeles una coalición denominada de ciudades de bienvenida. Estas ciudades estaban convencidas de que exponer y dar a conocer las contribuciones económicas y culturales de los inmigrantes a las ciudades, podría incrementar el apoyo a las políticas santuario (Huang y Liu, 2016).

La creciente criminalización de las minorías ha afectado a los latinos y la relación entre los inmigrantes y la policía local es un tema delicado en Chicago. La ciudad ha sido presionada interna y externamente para reducir su tasa de crímenes violentos. Por ejemplo, el 24 de enero de 2017, Trump twitteó: "Si Chicago no arregla la horrible 'carnicería', 228 tiros en 2017 con 42 asesinatos (un 24\% más que en 2016), enviaré el Feds [jerga para agencias federales de cumplimiento de la ley]" (Trump, 2017). Esto en una clara respuesta al alcalde de Chicago, Emmanuel Rahm, quien esa semana reforzó las ordenanzas de bienvenida y presentó una demanda en un tribunal de distrito para proteger los ordenamientos santuario.

La presión de los inmigrantes organizados de Chicago para expandir la protección de las políticas santuario de la ciudad llevó a la introducción del Trust Act en el gobierno de Illinois aún bajo el dominio republicano. El caso de Chicago demuestra el potencial de escalabilidad de las leyes y ordenanzas santuario cuando estas son parte de una estrategia más amplia que incluye políticas de incorporación y están ligadas al activismo político de los inmigrantes en la localidad. Como una activista de California explica: "Lo que está pasando ahora no es solamente un movimiento santuario. Es una estrategia moderna orientada a la comunidad que tiene un 
gran potencial para empoderar y prevenir las violaciones a los derechos civiles" (activista II, 2017).

\section{El futuro de los santuarios para inmigrantes: Escalabilidad y expansión de las prácticas}

En el estudio de las políticas públicas, la escalabilidad se refiere al potencial de un conjunto de políticas dinámicas para expandirse horizontal o verticalmente. En el primer caso siendo adoptadas por otras localidades o complementadas mediante ampliaciones en la ciudad de origen, y en el segundo caso cuando se reconoce su éxito y se adoptan a nivel estatal o federal.

La escalabilidad de las políticas santuario a nivel estatal (vertical) es considerada como un ejemplo modelo de transferencia de políticas públicas bottom-to-top que puede hacer frente a la política federal antiinmigrante llenando los vacíos que las jurisdicciones locales no pueden cubrir. Actualmente Vermont, Rhode Island, Nueva Jersey, Illinois, Nuevo México, Oregón y California tiene ordenanzas estatales que indican que en todo el estado está prohibido que la policía local intervenga en actividades de reforzamiento migratorio con ICE. Sin embargo, por la naturaleza de sus prácticas y el contenido de sus leyes, solo hay dos estados que pueden ser considerados como estados santuario: Illinois y California.

Como anteriormente se ha mencionado, un problema de las ciudades santuario es que por cuestiones estructurales tienen altas tasas de crimen y para los críticos de las ordenanzas santuario, las limitantes que se imponen a los policías al entrar en contacto con poblaciones de estatus migratorio mixto son un detrimento a la seguridad pública. Como consecuencia, las ordenanzas locales frecuentemente incluyen muchas excepciones bajo las cuales la policía puede verificar el estatus migratorio de un detenido y reportarlo a ICE.

Por ejemplo, en Chicago una excepción es en caso de sospecha de felonía, el problema es que bajo la ley estatal, usar documentos falsos puede ser considerado un delito de ese tipo. Frecuentemente, los migrantes indocumentados tienen que usar números de seguridad social falsos para trabajar ante la falta de mecanismos legales para regularizar su estancia en el país. Esta situación ha llevado a los impulsores de las leyes santuario a analizar como el solapamiento de jurisdicciones local-estatal provoca vacíos legales que limitan los efectos de las leyes de las ciudades santuario.

La presión de los inmigrantes organizados de Chicago para expandir la protección de las políticas santuario de la ciudad llevó a la introducción en 2018 del Trust Act en el congreso de Illinois aún bajo el dominio republicano. El Trust Act fue el resultado de las movilizaciones de los grupos de activistas comunitarios en Chicago y en el capitolio de Illinois. Las organizaciones recopilaron firmas y organizaron bancos telefónicos en apoyo de la iniciativa. Líderes latinos de Chicago llevaron a cabo actividades de Lobby para convencer a los representantes de otras localidades de los beneficios del Illinois Trust Act. Los debates legislativos, las votaciones y la firma incluso fueron transmitidos en vivo a través de las redes sociales de los migrantes.

La Illinois Trust Act (2017) prohíbe investigar, revisar, detener o arrestar a cualquier persona en Illinois teniendo como sospecha principal que es un inmigrante indocumentado. Establece que ninguna autoridad estatal colaborará en refuerzos 
migratorios. También se prohíbe la construcción de nuevos centros de detención migratoria en el estado. Asimismo, se incluye una cláusula para prevenir el uso de fondos locales para actividades de reforzamiento migratorio.

Incluso en 2018, los esfuerzos conjuntos y la movilización masiva de los inmigrantes en Chicago llevaron a la introducción de dos leyes complementarias aún más poderosas. La Safe Zones Act (2017) que prohíbe a nivel estatal que ICE operara en zonas denominadas sensibles como oficinas públicas, bibliotecas, escuelas y hospitales. La Anti-Registry Program Act (2018) que prohíbe la creación de listas y bases de datos basadas en el origen étnico y el estatus migratorio en el estado.

Otro de los casos más importantes de estado santuario es California, dos cuestiones lo hacen relevante, un proceso histórico y uno contemporáneo. El histórico nos remite a ver como California pasó de ser un estado conservador a uno progresista en unas cuantas décadas y esto se ve reflejado en su política migratoria. La impronta antiinmigrante de la Propuesta 187 en 1994, se ha borrado por el activismo migrante y la incursión de los latinos en la política formal a lo largo y ancho del estado. No obstante, la dinámica actual refleja los contrastes sobre la política migratoria local entre los condados, mientras que hay condados como Orange y Anaheim que han pasado resoluciones restrictivas para disuadir el asentamiento de los migrantes en esas ciudades, por otro lado encontramos grandes metrópolis como San Francisco, Los Ángeles, Oakland y Santa Ana que han expandido y defendido en cortes federales sus ordenanzas santuarios. Estas grandes ciudades santuario se han impuesto a los condados antiinmigrantes y lograron que California se convirtiera en un estado santuario.

En 2013, California pasó la primera Trust Act estatal, esta primera legislación dejaba sin efecto cualquier legislación que obligara a los gobiernos locales a cooperar forzosamente con las actividades de refuerzo migratorio, así como cualquier ley que obligara a los gobiernos locales a destinar recursos a perseguir inmigrantes. Esta provisión permitió que numerosas ciudades en California pasaran leyes santuario tras la elección de Donald Trump. De acuerdo con los jefes de la policía de Santa Ana, "La ley federal de inmigración actual va a causar un deterioro de los lazos con la comunidad extranjera, lazos que han llevado mucho tiempo y esfuerzo construir" (conferencia de prensa, Ayuntamiento de los Santa Ana, nota de campo, septiembre de 2016).

Es en 2018 cuando se publica la ley Santuario de California (California Values Act, 2017) en la que se establecen como zonas seguras para los inmigrantes a las escuelas, las cortes y los hospitales, es decir, ICE no puede operar en ninguno de estos espacios considerados socialmente sensibles. La Ley de los Valores de California también prohíbe expresamente el uso de recursos locales en refuerzo migratorio, la policía local solo puede cooperar en investigaciones federales de tráfico de personas, de drogas y cuestiones de ciberseguridad. La ley santuario de California es la que más oposición ha generado, incluso sus opositores colocaron señalizaciones falsas en las fronteras del estado que decían "Bienvenidos a California. Oficialmente estado santuario. Criminales, ilegales y maras son bienvenidos. Los demócratas necesitan los votos".

A diferencia de Illinois, el estado santuario de California restringe a las localidades de cooperar con ICE, no permite la autonomía de sus ciudades. El caso de Illinois también incluye mayores excepciones en casos criminales, mientras que California no permite cooperación policial cuando el objetivo final implique aplicar leyes de 
inmigración. Por su parte, la fortaleza de Illinois reside en la prohibición de hacer listas y bases de datos basados en el estatus migratorio y la raza. Sin duda, ambos casos ilustran como el espectro de políticas santuario puede llevarse a los extremos de oponerse a la política federal pese a la amenaza de ser llevados a cortes, de no dejar huecos en las protecciones y de cambiar totalmente la relación de la policía con las comunidades de estatus mixto.

El caso de los estados santuario ilustra la futura escalabilidad vertical de las estrategias para limitar el efecto de la política antiinmigrante sobre la vitalidad de las localidades. Pero también se ha presentado la escalabilidad horizontal a través de la expansión de las ciudades santuario a nuevos horizontes. El tipo de ordenanzas y políticas que actualmente llevan la vanguardia son la creación de santuarios digitales. Este caso se refiere al uso responsable de la información, sobre todo aquello que implique la captación de datos sensibles biométricos y del estatus legal.

Los activistas migrantes y los gobiernos locales se han dado cuenta que hay información sensible de los migrantes a la que ICE puede acceder que ha sido recogida por la ciudad para trámites de identificación, servicios educativos, servicio de salud, entre otros. Oficialmente ICE obtiene datos de los depósitos de fuerzas del orden locales, del departamento de vehículos motorizados, de los lectores de matrículas y del registro de visas de trabajo y de estudiantes. Las leyes de protección de datos personales de Estados Unidos mencionan que en el caso de extranjeros puede haber triangulación entre agencias para juntar la información. Estas prácticas han dado paso a la presión local para crear legislaciones y políticas sobre santuarios digitales.

En 2017 en Chicago, un inmigrante indocumentado llamado Wilmer Catalán fue acusado de ser miembro de pandillas, los oficiales de inmigración tomaron sus datos y su estatus migratorio de una base de datos de la ciudad de Chicago. ${ }^{7}$ Una cuestión de simple asociación y de registro inadecuado causó que ICE lo enlistara como un migrante de alta prioridad, por lo que pidió a la policía local su apoyo y la detención se dio de forma violenta en su domicilio. En el caso de Chicago, los activistas presionaron al gobierno de la ciudad para exigir a ICE y a la policía una explicación de los criterios para crear bases de datos de criminales y pandillas, ya que se encontró que en California e Illinois, incluso niños de dos años estaban en dichas bases (California State Auditor, 2016). Como respuesta a este tipo de episodios, en 2017 la ciudad de Portland cedió a la presión de los activistas y accedió a borrar todas sus bases de datos de pandillas ya que se reconoció que no hay una metodología exacta ni pruebas contundentes para incluir a las personas allí.

Simultáneamente, los activistas migrantes aprendieron de la amplia participación en el programa DACA, que el facilitar información personal a los gobiernos puede ser un arma de doble filo ante la poca incertidumbre sobre la continuidad de los programas (Cruz, 2016). En esta misma línea, los líderes inmigrantes denunciaron que hay un sentir de decepción en sus comunidades sobre los programas públicos que se ofertan para reducir las desventajas de los migrantes indocumentados como las identificaciones municipales.

\footnotetext{
${ }^{7}$ Según el Chicago Tribune, los criterios que la policía de Chicago usa para incluir a un individuo en la base de datos de pandillas son tatuajes y uso de símbolos asociados con las pandillas, familiaridad (apellidos), asociación (residencia) y otros aspectos subjetivos (Serrato, 2017).
} 
Por ejemplo, la Coalición de Illinois por los Derechos de los Migrantes y los Refugiados denunció que los migrantes no quieren aplicar para programas como las tarjetas de identificación municipal o incluso naturalizarse obteniendo la ciudadanía debido a que ellos consideran que con esto se podría obtener acceso a las bases de datos de los programas públicos. Como respuesta, un Concejal Latino de Chicago explicó en entrevista:

Le recomiendo a todos los residentes que apliquen al programa Municipal ID. Estamos evaluando opciones para destruir las bases de datos; aunque actualmente no podemos asegurar que la ciudad pueda legislar sobre los santuarios digitales. Sin embargo, el gobierno de la ciudad de Chicago se va a hacer responsable por la protección de datos de todos sus residentes (Cárdenas, 2017).

Los activistas piden que se siga la tendencia de la ciudad santuario de Nueva York donde se tiene la práctica de destruir los registros individuales y anonimizar la base de datos central, que además está encriptada.

Entonces, aunque no tenemos un caso concreto que pueda ser identificado como un santuario digital, la tendencia de las políticas santuario se dirige hacia allí. Hay dos avances en la materia, en primer lugar, se encuentran las prohibiciones de hacer registros basados en la raza, el estatus migratorio o la religión que actualmente están vigentes en todo el estado santuario de Illinois y en la ciudad santuario de San Francisco. La segunda cuestión es el manejo responsable de datos de programas, por ejemplo las identificaciones municipales en el caso de la ciudad de Nueva York.

Las ciudades presionadas por sus organizaciones civiles siguen buscando formas de proteger a los inmigrantes en contraposición a la postura federal antiinmigrante de la actualidad. Recientemente se presentó un episodio que sirvió como aliciente para la discusión sobre los límites de las protecciones santuarios, un activista explicó:

Recientemente la alcaldesa de Oakland alertó a los residentes de su ciudad de una operación de ICE en el norte de California, lo que probablemente imposibilitó unas 800 detenciones. Eso es lo menos que esperamos de nuestros representantes, queremos que ellos compartan la información que tengan con la comunidad (activista III, 2017).

Estas dinámicas ilustran el rumbo incierto que seguirán las políticas santuario, ya que se reproducen frente a contextos de hostilidad política. Es decir, una reforma migratoria federal comprehensiva probablemente volvería innecesarias las políticas santuario; sin embargo, la tendencia política parece ir en sentido contrario, por lo que la escalabilidad vertical y horizontal sigue siendo viable para las ciudades proinmigrante.

\section{Conclusiones}

Al analizar las prácticas de las ciudades santuarios más representativas descritas en este artículo, podemos observar varias tendencias. Para recapitular el estudio cross caso, primero se retoman los aspectos procedimentales de la relación con 
ICE, posteriormente se analizan las prácticas locales y finalmente las políticas de inmigración.

Tanto los santuarios de retórica, los informales y los de bienvenida no tienen suscritos acuerdos con ICE (Department of Homeland Security, 1996, sección 287g), lo cual ha permitido a las ciudades ampararse cuando los estados pretenden presionarlas para cooperar en el reforzamiento migratorio, esto también ha sido útil para ganar batallas legales. Por ejemplo, en julio de 2018 seis estados y la ciudad de Nueva York introdujeron una querella en contra de las amenazas de Trump de prohibir fondos anticrimen (Bolsas Byrne) para aquellas ciudades que se rehusaran a cooperar con ICE (Robbins, 2018). Igualmente, la novena corte de distrito de apelaciones falló a favor de las ordenanzas santuario de San Francisco y Santa Clara contra el Departamento de Justicia (Thanawala, 2018).

Un aspecto importante en la relación con ICE es que la policía local, en ciudades con leyes santuario, no reporta el estatus migratorio de los detenidos, en el caso de los santuarios de facto se establecen plazos mínimos y mediante retrasos burocráticos frecuentemente no se hacen efectivos lo anuncios. Otro común denominador es que los tres casos contienen múltiples excepciones, todas relacionadas con la seguridad pública derivadas de las altas tasas de crimen que por cuestiones estructurales y no exclusivas de la migración tienen estas ciudades. Con respecto a las prácticas locales de gestión migratoria, las ciudades santuario tienen la práctica de no recolectar el estatus migratorio, los santuarios de acogida lo prohíben determinantemente, los santuarios de retórica lo hacen en algunas circunstancias, los santuarios informales no tienen protocolos al respecto pero tampoco tienen motivación para hacerlo sistemáticamente.

Ahora bien, las cuestiones que implican reformas estructurales y dinámicas de justicia distributiva son las que tienden a quedarse en el plano discursivo en los santuarios de retórica y no son parte de los santuarios de facto.

La cuestión del acceso justo y universal a los servicios de la ciudad para todos los residentes sin excepción es una arista de contraste. Los santuarios de facto están limitados por su contexto, si no se pueden hacer reformas procedimentales es menos probable que lleven a cabo reformas de justicia distributiva a favor de los inmigrantes. Los santuarios de retórica establecen cláusulas de acceso igualitario pero no hay capacidad estructural para que se lleve a cabo ya que no van acompañadas de presupuesto justo. En cuanto a las ciudades de bienvenida, la adopción de leyes santuario se acompañó de políticas de instrumentalización y de políticas de acogida como la financiación de asistencia legal, campañas "conozca sus derechos" y talleres de ciudadanía. Sin embargo, su efectividad dependerá tanto de la capacidad de los activistas para presionar por rendición de cuentas y presupuesto justo, como del clima político dominante.

Las dinámicas evidenciadas por los casos anteriores han funcionado para evaluar las hipótesis que guiaron esta investigación y que además nos sirven para exponer las conclusiones de este estudio:

Primero, las motivaciones de los inmigrantes coinciden en la misión de mediar los efectos de las políticas antiinmigrantes de otros niveles de gobierno y de exponer el carácter progresista de la política local; sin embargo, su contenido e instrumentalización difieren en sus efectos prácticos sobre la seguridad pública y la incorporación de los migrantes. 
Segundo, la naturaleza de las políticas santuario se debe en gran parte al tipo de presión que el activismo local ejerce en favor de su adopción y expansión, ya que los grupos hacen notar lo que se está haciendo en otros espacios y exponen las fallas de las prácticas actuales.

Tercero, el análisis de las trayectorias políticas permitió rastrear las dinámicas detrás de las políticas santuario con mayor grado de insurgencia. Las prácticas santuario más vanguardistas e insurgentes están ligadas a los movimientos locales, pero también a defender su acceso a fondos y proteger su autonomía política, esto con el objetivo de mediar las prioridades y agendas tanto de sus poblaciones nativas como de sus minorías migrantes.

\section{Referencias}

Bauder, H. (2016). Sanctuary Cities: Policies and Practices in International Perspective. International Migration, 55(2), 174-187. https://doi.org/10.1111/imig.12308

Bender, S. y Arrocha, W. (Eds.). (2017). Compassionate Migration and Regional Policy in the Americas. Londres, Inglaterra: Palgrave Macmillan.

Bilke, C. (2009). Divided We Stand, United We Fall: A Public Policy Analysis of Sanctuary Cities' Role in the "Illegal Immigration" Debate. Indiana Law Review, 42(1), 165-193.

California State Auditor. (2016). The CalGang Criminal Intelligence System. Recuperado de https://www.auditor.ca.gov/pdfs/reports/2015-130.pdf

Cárdenas, G. (Mayo de 2017). Voces de migrantes [archivo de video]. Recuperado de https: / /www.youtube.com/watch?v=6Ga22GaZ5ig

Chand, D. E. y Schreckhise, W. D. (2015). Secure Communities and Community Values: Local Context and Discretionary Immigration Law Enforcement. Journal of Ethnic and Migration Studies, 41(10), 1621-1643. https://doi.org/10.1080/1369 183X.2014.986441

Cook, M. L. (2013). ls Incorporation of Unauthorized Immigrants Possible? Inclusion and Contingency for Nonstatus Migrants and Legal Immigrants. En J. Hochschild, J. Chattopadhyay, C. Gay y M. Jones-Correa (Eds.), Outsiders no More? Models of Immigrant Political Incorporation (pp. 43-63). Nueva York, Estados Unidos: Oxford University Press.

Cornelius, W. A. (2010). Introduction. En M. W. Varsanyi (Ed.), Taking Local Control: Immigration Policy Activism in U.S. Cities and States (pp. vii-viii). Standford, California: Standford University Press.

Cruz, E. (2016). Young Immigrants Association and the Future Latino Leadership in the United States: Dreamer's Social Capital and Political Engagement. Norteamérica, 11(2), 165-192. https://doi.org/10.20999/nam.2016.b007

Cunningham, C. (2017). Sanctuary Lost? Exposing the Reality of the "Sanctuary-City" Debate \& Liberal States-Rights' Litigation. Wayne Law Review, 63(2) 1-72.

Department of Homeland Security. (1996). Delegation of Immigration Authority Section 287(g) Immigration and Nationality Act. Recuperado de https://www.ice.gov/287g

Eisinger, P. (1998). City Politics in an Era of Federal Devolution. Urban Affairs Review, 33(3), 308-325. https://doi.org/10.1177/107808749803300304 
Filomeno, F. A. (2017). Theories of Local Immigration Policy. Reino Unido: Palgrave Macmillan.

Glick, N. y Chaglar, A. (2009). Towards a Comparative Theory of Locality in Migration Studies: Migrant Incorporation and City Scale. Journal of Ethnic and Migration Studies, 35(2), 177-202. https://doi.org/10.1080/13691830802586179

Gonzalez, B., Collingwood, L. y El-Khatib, S. O. (2017). The Politics of Refuge: Sanctuary Cities, Crime, and Undocumented Immigration. Urban Affairs Review, 1-38. https://doi.org/10.1177/1078087417704974

Harwood, S. y Myers, D. (2002). The Dynamics of Immigration and Local Governance in Santa Ana. Policy Studies Journal, 30, 70-91. https://doi. org/10.1111/j.1541-0072.2002.tb02130.x

Hoekstra, M. S. (2017). Governing Difference in the City: Urban Imaginaries and the Policy Practice of Migrant Incorporation. Territory, Politics, Governance, 6(3), 1-19. https://doi.org/10.1080/21622671.2017.1306456

Hopkins, D. J. (2010). Politicized Places: Explaining Where and When Immigrants Provoke Local Opposition. American Political Science Review, 104(1), 40-60. https:// doi.org/10.1017/S0003055409990360

Huang, X. y Liu, C. Y. (2016). Welcoming Cities: Immigration Policy at the Local Government Level. Urban Affairs Review, 54(1), 3-32. https://doi. org/10.1177/1078087416678999

Kent, S. L. y Carmichael, J. T. (2017). Municipal Law Enforcement Policy on Illegal Immigration Stops: Do Social Factors Determine how Aggressively Local Police Respond to Unauthorized Immigrants? Sociological Inquiry, 87(3), 421-448. https://doi.org/10.1111/soin.12155

Levine, J. R. y Gershenson, C. (2014). From Political to Material Inequality: Race, Immigration, and Requests for Public Goods. Sociological Forum, 29(3), 607-627. https://doi.org/10.1111/socf.12106

Lieberman, R. C. (2013). Ideas and Institutions in Immigrant Political Incorporation. En J. Hochschild, J. Chattopadhyay, C. Gay y M. Jones-Correa (Eds.), Outsiders no More? Models of Immigrant Political Incorporation (pp. 82-94). Nueva York, Estados Unidos: Oxford University Press.

Lyons, C., Velez, M. B. y Santoro, W. A. (2013). Neighborhood Immigration, Violence, and City-Level Immigrant Political Opportunities. American Sociological Review, 78(4), 604-632. https://doi.org/10.1177/0003122413491964

Mollenkopf, J. (2013). Dimensions of Immigrant Political Incorporation. En J. Hochschild, J. Chattopadhyay, C. Gay y M. Jones-Correa (Eds.), Outsiders no More? Models of Immigrant Political Incorporation (pp. 107-118). Nueva York, Estados Unidos: Oxford University Press.

Paik, A. N. (2017). Abolitionist Futures and the US Sanctuary Movement. Race E्F Class, 59(2) $1-23$.

Partnership for a New American Economy. (Agosto de 2016). The Contributions of New Americans in Colorado. Recuperado de http://research.newamericaneconomy. org/wp-content/uploads/2017/02/nae-co-report.pdf

Passel, J. S. y Cohn, D. (2017). 20 metro areas are home to six-in-ten unauthorized immigrants in U.S. Pew Research Center. Recuperado de http://www.pewresearch.org/facttank/2017/02/09/us-metro-areas-unauthorized-immigrants/ 
Paxton, K. (Diciembre de 2017). [Conferencia de prensa]. San Antonio, Estados Unidos.

Ramakrishnan, K. S. y Wong, T. K. (2007). Immigration Policies go Local: The Varying Responses of Local Governments to Undocumented Immigration. University of California, Riverside.

Ridgley, J. (2008). Cities of Refuge: Immigration Enforcement, Police, and the Insurgent Genealogies of Citizenship in U.S. Sanctuary Cities. Urban Geography, 29(1), 53-77. https://doi.org/10.2747/0272-3638.29.1.53

Robbins, L. (18 de julio de 2018). New York City and State File Suit Over Grants Linked to ICE Cooperation. Recuperado de https://www.nytimes.com/2018/07/18/nyregion/ sanctuary-new-york-city-state-lawsuit-immigration.html

Saldaña, R. (Septiembre de 2018). [Conferencia de prensa]. San Antonio, Estados Unidos.

San Francisco Approves Bill to Designate it a Sanctuary (24 de diciembre de 1985). New York Times. Recuperado de https://www.nytimes.com/1985/12/24/us/san-francisco-approves-bill-to-designate-it-a-sanctuary.html

Schiller, N. G., Caglar, A. y Guldbrandsen, T. C. (2006). Beyond the Ethnic Lens: Locality, globality, and Born-again Incorporation. American Ethnologist, 33(4), 612-633. https://doi.org/10.1525/ae.2006.33.4.612

Serrato, J. (6 de diciembre de 2017). Chicago Tribune Admits Gang Database Error that Enabled ICE Raid. The Chicago Tribune. Recuperado de https://www.chicagotribune.com/hoy/ct-chicago-police-admits-gang-database-error-20171206-story.html

Sessions, J. (2017). Attorney General Sessions Delivers Remarks on Sanctuary Policies. Estados Unidos: The United States Department of Justice.

Steil, J. P. y Vasi, I. B. (2014). The New Immigration Contestation: Social Movements and Local Immigration Policy Making in the United States, 2000-2011. American Journal of Sociology, 119(4), 1104-1155. https://doi.org/10.1086/675301

Stoltz, N., Hamilton, N. y Loucky, J. (2009). The Sanctuary Movement and Central American Activism in Los Angeles. Latin American Perspectives, 36(6), 101-126. https://doi. org $/ 10.1177 / 0094582$ X09350766

Thanawala, S. (1 de agosto de 2018). Court Strikes Down Trump Push to Cut 'Sanctuary City' Funds. The Associate Press. Recuperado de https://www.bloomberg.com/news/ articles/2018-08-01/urgent-us-appeals-court-trump-sanctuary-cities-order-is-illegal

Trump, D. (24 de enero de 2017). If Chicago doesn't fix the horrible "carnage" going on, 228 shootings in 2017 with 42 killings (up 24\% from 2016), I will send in the Feds! Recuperado de https://twitter.com/realdonaldtrump/status/824080766288228352

United States Citizenship and Immigration Services. (2019). Estatus de Protección Temporal. Recuperado de https://www.uscis.gov/es/TPS

Varsanyi, M. W. (2011). Neoliberalism and Nativism: Local Anti-Immigrant Policy Activism and an Emerging Politics of Scale. International Journal of Urban and Regional Research, 35(2), 295-311. https://doi.org/10.1111/j.1468-2427.2010.00958.x

Varsanyi, M. W., Lewis, P. G., Provine, D. M. y Decker, S. (2011). A Multilayered Jurisdictional Patchwork: Immigration Federalism in the United States. Law and Policy, 34(2), 138-158. https://doi.org/10.1111/j.1467-9930.2011.00356.x 


\section{Entrevistas}

Activista I. (Abril de 2017). Entrevista de Estefanía Cruz Lera/ activista de Chicago [transcripción], Coalición de Illinois por los Derechos de los Inmigrantes y los Refugiados, Chicago, Estados Unidos.

Activista II. (Agosto de 2017). Entrevista de Estefanía Cruz Lera/ activista [transcripción], Santa Ana, Estados Unidos.

Activista III. (Agosto de 2017). Entrevista de Estefanía Cruz Lera/ activista [transcripción], Los Ángeles, Estados Unidos.

Ramírez-Rosa, C. (Mayo de 2017). Entrevista de Estefanía Cruz Lera/ Concejal de Chicago [transcripción], Universidad Nacional Autónoma de México-Chicago, Estados Unidos.

\section{Material legislativo}

Anti-Registry Program Act. Asamblea General del Estado de Ilinois. 24 de agosto de 2018. Recuperado de http://www.ilga.gov/legislation/billstatus.asp?DocNum=3488\&GAID=14\&GA=100\&DocTypeID=SB\&LegID=111560\&SessionID=91

California Values Act (SB54). Senado del Estado de California. 5 de octubre de 2017. Recuperado de http://www.iceoutofca.org/ca-values-act-sb54.html

Illinois Trust Act. Asamblea General del Estado de Illinois. 28 de agosto de 2017. Recuperado de http://www.ilga.gov/legislation/ilcs/ilcs3.asp?ActID=2117\&ChapterID $=61$

New Americans Plan. Office of the New Americans, Chicago. 4 de diciembre de 2012.

Public Safety Enforcement Priorities Act, Denver City Code, 14 de julio de 2017.

Safe Zones Act. Asamblea General del Estado de Ilinois. 1 de diciembre de 2017. Recuperado de http://www.ilga.gov/legislation/BillStatus.asp?DocTypeID=HB\&DocNum=426\&GAID=14\&SessionID=91\&LegID=99918

Texas Senate Bill 4 (SB4), Texas Capitol. (15 de noviembre de 2016). Recuperado de https://capitol.texas.gov/tlodocs/85R/billtext/pdf/SB00004I.pdf

The United States Department of Justice (USDJ). (2017). Justice Department Sends Letters to 29 Jurisdictions Regarding Their Compliance with 8 U.S.C. 1373. Recuperado de https://www.justice.gov/opa/pr/justice-department-sends-letters-29-jurisdictions-regarding-their-compliance-8-usc-1373

United States Congress. (2001). United States of America: Uniting and Strengthening America by Providing Appropriate Tools Required to Intercept and Obstruct Terrorism Act of 2001 (USA Patriot Act). Recuperado de https://www.refworld.org/docid/3dea43144. html

Welcoming Ordinances. Chicago City Council. 13 de septiembre de 2012. 


\section{Estefanía Cruz Lera}

Mexicana. Doctora en Ciencias Políticas, Política Pública y Relaciones Internacionales por la Universitat Autónoma de Barcelona. Maestra y Licenciada en Estudios Internacionales por la Universidad Nacional Autónoma de México (UnAm). Actualmente se desempeña como investigadora de tiempo completo (SIJA) en el Centro de Investigaciones sobre América del Norte de la unAm. Líneas de investigación: incorporación de minorías políticas y sistema político estadunidense. Entre sus publicaciones recientes: Cruz, E. (2019). La gobernanza de las migraciones transnacionales china y mexicana hacia Estados Unidos: Los nexos entre la Diáspora y el Estado creativo para el codesarrollo. Revista Mexicana de Ciencias Políticas y Sociales, 64(236), 21-48. 